\title{
Automatic Mobile Photo Tagging Using Context
}

\author{
Ke Huang, Xiang Ding, Guanling Chen, Kate Saenko \\ Department of Computer Science, University of Massachusetts Lowell \\ \{khuang, xding, glchen, saenko\}@cs.uml.edu
}

\begin{abstract}
The market of smartphones has been exploding, and taking pictures is a basic, maybe one of the most important functions of a smartphone. In this paper we address the problem of managing a large amount of mobile photos by automatically tagging the photos, so they can be easily browsed or searched later. Unlike other content-based photo tagging approaches, this paper's main contribution is to explore an alternative opportunity of automatic photo tagging using contextual information. Both clustering and similarity-based approaches were studied for photo tagging using context such as date, time, location, environment noise, and human faces. The results show that there are intrinsic connections between contextual information and photo tags, and similarity-based approach outperforms clustering-based tagging significantly.
\end{abstract}

Keywords-Context Awareness, Photo Tagging, Smartphone Sensing, Face Recognition

\section{INTRODUCTION}

Nowadays digital photos and videos continue to grow rapidly, especially with the proliferation of high quality digital cameras embedded in mobile devices. With these devices, people are able to take photos anytime and anywhere they want. This brings with it the demand of personal photo management. Tagging photo with keywords has proved to be an efficient and successful approach to facilitate the photo management. Most users, however, either do not have time to tag the photos on the spot or do not want to make the manual effort of labeling a large amount of photos.

To address this problem, automatic photo tagging has been introduced, which unfortunately has three limitations when used for personal photo management. First, most of autotagging approaches are based on the analysis of picture content while existing algorithms still have difficulty to recognize objects with high accuracy. Second, there is no way for the algorithms to determine meaningful tags that are personal in nature, such as the names of the people in the photo. Finally, users may tag their photos with labels not shown in the picture, such as the event where the photo was taken.

In this paper we explore an alternative approach that leverages contextual information for photo tagging. Our hypothesis is that the pictures share similar context may also share some tags. There are some latent factors, which can be represented by four 'W's (When, Where, What, and Who), bridging the contextual information and photo tags (Figure 1). For example, a photo of white house taken during the labor day holiday can be automatically tagged with "white house" and "labor day" if there are existing photos with these tags sharing similar context, taken either by the same user or by others.

To validate our approach of context-based photo tagging, we developed an Android application that can take a photo and

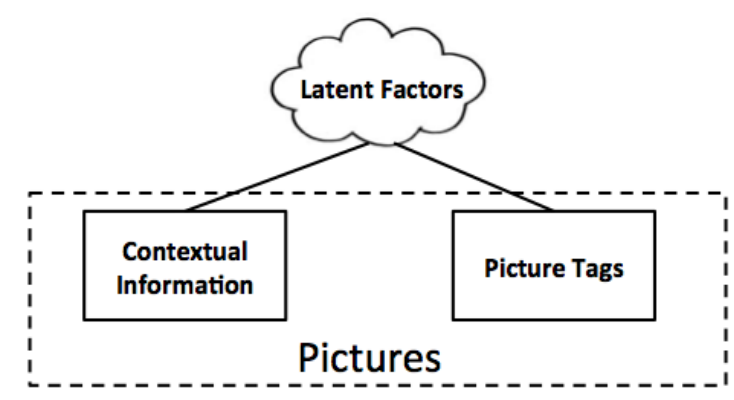

Fig. 1. Contextual Information and Tags

capture its context simultaneously. Currently, we collect four types of contextual information: when (date and time), where (GPS, WiFi SSIDs), what (noise) and who (face detected). The idea is then to develop an algorithm to automatically recommend tags chosen from existing tag set of the photos sharing similar context. As a user continues to use the proposed system and as more users collaboratively tag the photos, we expect personal photo management will be much easier with significantly reduced manual effort.

\section{RELATED WORK}

Image tagging has been a topic of extensive research given its applications to image retrieval [1], [2]. Several researchers have been working on creating metadata for mobile photos. In [3], [4], Sarvas et al. automate the creation of photo content metadata leveraging automatically available contextual metadata (spatial, temporal, and social, i.e., "where", "when", and "who") on the mobile phone. It generates more metadata for the captured photo based on the metadata of "similar" photos on the server. However, their contexts are rather limited and have coarse-granularity, as only location and pixel data are considered in generating metadata. In [5], the authors proposed an approach that computes event and location groupings of photos based on their time and location. As users tag the people in their collection, patterns of re-occurrence and cooccurrence of different people in different locations and events are expected to emerge. The system uses these patterns to assist users in tagging photos by suggesting a short list of candidates. Different from our work, they focus on suggesting labels for identities (people). TagSense [6] is a mobile phone based collaborative system that uses out-of-band sensing to sense the people, activity and context (when, where) in a picture and merges them to create tags on-the-fly. The tags are organized into a "when-where-who-what" format. However, the tags generated are predefined and tags from other related photos cannot be effectively used. 


\section{APPROACH}

The main idea of the proposed work is to use clustering techniques and leverage context as features to cluster and autotag photos. Figure 2 is an overview of the work which is divided into two stages. The first stage is data collection by an Android application we developed. The second stage is data analysis which leverages these contextual information to infer tags for newly taken photos. We only focus on the second stage in this paper and we consider both clustering-based and similarity-based approaches.

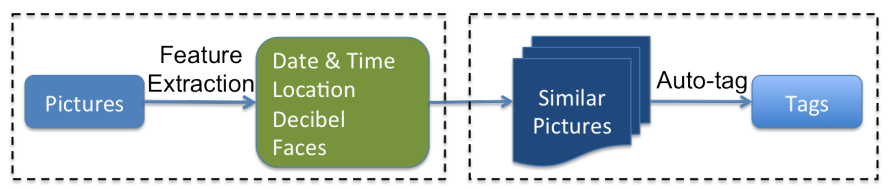

Fig. 2. Overview of the Approach

\section{A. Feature Extraction}

The contextual information we consider including temporal, spatial, environmental and participants information. Each photo is associated with several context records which will be converted into feature vectors. The detailed description of the contextual information and how to convert them into feature vectors are described in following sections.

1) Date and Time: The most common and widely used contextual information is date and time. We also consider that there are some temporal patterns when a user is taking a picture. These patterns can be divided into two categories in two scales. The first pattern scales in year, month and day which is a long term pattern embedded in photos. Examples are the photos that are taken at some annual events like Christmas day or someone's birthday. The second pattern scales in hour of day which is a short term pattern. This pattern is not general to most of users though. For some photos this could be an important pattern since these photos can only be taken in a specific time slot. The sunset pictures, for instance, can only be taken between 5PM and 8PM in most of areas. This temporal contextual information is represented by a 4-dimension feature vector $F_{T}$ :

$$
F_{T}=[\text { year, month, day, hour }]
$$

2) Location: The location information tells the story of where a photo was taken. If a place is significant to a user, there may be many photos taken in that place, since an activity or event does not happen in a random place. We have collected two kinds of location context, the WiFi SSIDs scanned by the phone and the GPS information. We do not focus on a precise location but whether the location is significant or not. The spatial feature vector $F_{L}$ is defined by vector 2 .

$$
F_{L}=\left[V^{S S I D}, \text { latitude, longitude }\right]
$$

In this definition, there is an SSID indicator vector $V^{S S I D}$. The dimension of $V^{S S I D}$ is the number of all SSIDs in the dataset, and the value of an entry is 1 if that SSID was discovered when taking the photo, otherwise it is 0 . The geo-information is set to -999 if GPS is not available. If two pictures were taken within one minute, the later picture will use the previous one's location context. There are two advantages of doing this. First of all, it saves power consumption. Secondly, we only focus on the location that is significant to a user and it is very unlikely that a user will go to another significant place within one minute.

3) Acoustic: The noise context is an effective measure of the surrounding environment and can be sensed by the phone. It is used to indicate the scenario of a picture. A noisy environment is more likely to have some event or activity, even no person in the photo. On the other hand, a photo that was taken in a quiet environment is more likely to be a landscape picture. It is represented by a one-dimension vector $F_{E}$.

$$
F_{E}=[\text { decibel }]
$$

The decibel value is obtained by recording and analyzing a 3 seconds environment sound when taking the picture. The extraction of noise context is also done in background which will not affect user to take another picture. If two picture were taken within one minute, the noise context of these two will set to be same in order to save power consumption. The idea behind this mechanism is that a user will not move far away within one minute and these two pictures should be taken in the same scenario.

4) Faces: Since it is hard to interpret the content of a picture, we choose to analyze who has participated in a photo instead. This is done by face detection and face recognition algorithms. Each face can be detected by the APIs which is provided by Android system effectively, and then, the face will be extracted from the raw image and a background process will handle the face recognition procedure. OpenCV provides multiple face recognition algorithms and APIs. In average, a face can be recognized by the smart-phone within 2 seconds. Since we were using the face recognition APIs provided by OpenCV, the recognition accuracy highly depends on the efficiency of the algorithms provided by OpenCV which is out of the discussion of this paper. For the face recognition procedure, a user needs to manually tag all faces at the very beginning to let the algorithm learn the face model. The face context vector $F_{P}$ is defined in equation 4:

$$
F_{P}=\left[\text { faceCount }, V^{\text {Face }}\right]
$$

The notation $V^{\text {Face }}$ is an indicator vector. We also added the number of faces in a photo as a context. The idea is that the number of participants indicates the type of the photo. For example, if only one face is in the photo that photo could be a portrait picture, while many faces indicate that the picture is about some special activity or event.

5) Feature Vector: We combine all these contextual information as a whole and the feature vector of a photo is defined by Equation 5 hereafter.

$$
F=\left[F_{T}, F_{L}, F_{E}, F_{P}\right]
$$

Since there are two indicator vectors $V^{S S I D}$ and $V^{\text {Face }}$, the feature vector is a sparse vector that brings a technical challenge to clustering photos.

\section{B. Clustering-based Tagging}

The first approach we studied is called clustering-based tagging. Since there are some latent factors bridging the 
contextual information and photo tags, the photos in the same cluster would share some common tags. We have no prior knowledge of the number of clusters, hence we compared two unsupervised clustering algorithms: Mean-Shift [7] and DBSCAN [8].

1) Mean-Shift [7]: The mean shift algorithm is a nonparametric clustering technique which does not require prior knowledge of the number of clusters, and does not constrain the shape of the clusters. This is an iterative method, and starts with an initial estimate $x$ (mean of cluster). Given a kernel function $K\left(x_{i}-x\right)$, the algorithm determines the weight of nearby points by iteratively re-estimating the mean of data points. The Gaussian kernel on the distance to the current estimate is used, $K\left(x_{i}-x\right)=e^{-c\left\|x_{i}-x\right\|^{2}}$, where $c$ is a parameter. Then the weighted mean of the density in the window determined by $K$ is defined:

$$
m(x)=\frac{\sum_{x_{i} \in N(x)} K\left(x_{i}-x\right) x_{i}}{\sum_{x_{i} \in N(x)} K\left(x_{i}-x\right)}
$$

where $N(x)$ is the neighborhood of $x$, a set of points for which $K(x) \neq 0$. Then the algorithm sets $x \leftarrow m(x)$, and repeats the estimation until $m(x)$ converges.

2) DBSCAN [8]: The DBSCAN algorithm is a densitybased algorithm. It can identify clusters in large spatial data sets by looking at the local density of database elements. DBSCAN's definition of a cluster is based on the notion of density reachability. A point $q$ is directly density-reachable from a point $p$ if it is not farther away than a given distance $\operatorname{eps}(\epsilon)$ and if $p$ is surrounded by sufficiently many points (min_points) such that one may consider $p$ and $q$ to be part of a cluster. $q$ is called density reachable from $p$ if there is a sequence $p_{1}, \ldots, p_{n}$ of points with $p_{1}=p$ and $p_{n}=q$ where each $p_{i+1}$ is directly density reachable from $p_{i}$. The relation of density reachable is not symmetric. $q$ might lie on the edge of a cluster, having insufficiently many neighbors to count as dense itself. This would halt the process of finding a path that stops with the first non-dense point.

\section{K-Neighbor Similarity-based Tagging}

The $K$-Neighbor similarity algorithm is adapted from the KNN algorithm but has nothing to do with classification. Just as shown in Figure 1, the photos that have similar contextual information also share similar tags. It follows that if we can find the nearest neighbors of a specific photo, we can infer tags for it from neighbors. For two distinct photos $x$ and $y$, we first get their feature vectors $F^{x}$ and $F^{y}$, respectively, and the distance between two is calculated by the Euclidean distance that is shown in Equation 7.

$$
D_{x y}=\left\|F^{x}-F^{y}\right\|
$$

Before we calculate the distance, all features are scaled to prevent some features from dominating the calculation. We calculate all distances and select $K$ neighbors that have the nearest distance. All tags of these neighbors are counted and the top used tags are recommended. This algorithm does not require clustering and can infer tags directly from its neighbors. In this paper, the $K$ value we chose is set to 5 with the consideration of the size of the dataset we have.

\section{DATASET}

Since there is no publicly available data set that contains the contextual information described in the above section, we built our data set by implementing an Android application. The data set in this work consists of 130 pictures. All pictures were taken within 4 days of different time slots at 4 different places. Among them, most of the photos are landscape photos, and there are 32 photos have participants. In total, there are 5 people and 35 faces have been detected. Hence, this data set covers all contextual information we need for the purpose of clustering or calculating the similarity distance. All 130 photos are manually tagged with at least 4 tags. The tags contain descriptions of the significant place name, events, activity, contents, and other special description like "spring". An image example is shown in Figure 3, and the tags of this image are ducks, bridge, Merrimack river, riverside, sunset and twilight. These photos are divided into training data set and testing data set. The training data set contains $70 \%$ of the pictures and the remaining $30 \%$ are for testing.

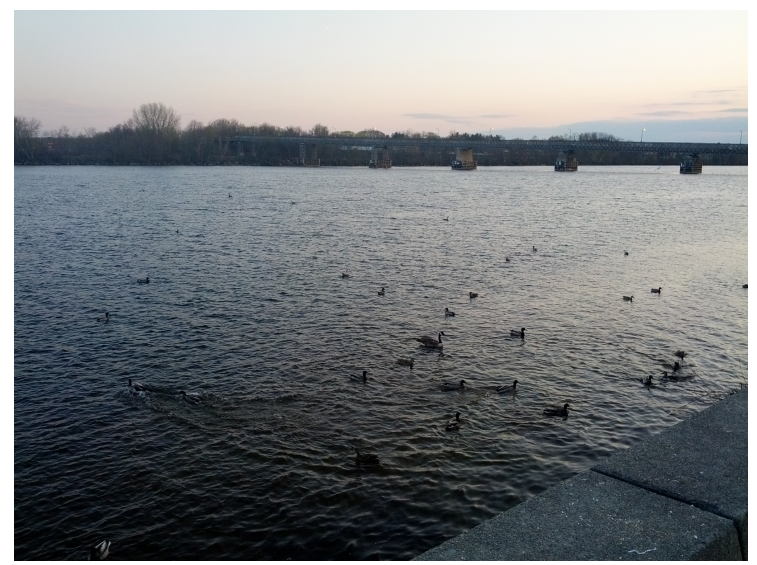

Fig. 3. An Image Example

\section{Evaluation}

The evaluation is conducted based on the two approaches we have proposed. Before applying our methods, the data set is shuffled and then divided into the training and testing data set. Since there is no existing literature that leverages contextual information to infer tags for photos, we introduce a naive baseline method which calculate the frequencies of all tags in the training data set, and then recommends the tags with highest frequency. The hit-rate or success-rate of recommendation is also introduced to measure the effectiveness of the recommendation. Let the tag set $\tau$ be the results of the recommendation, and set $\rho$ be the ground truth tag set, then the hit-rate is defined in Equation 8:

$$
H_{\tau}=\frac{|\tau \cap \rho|}{|\tau|}
$$

The hit-rate is obtained by running multiple times of the algorithm and then calculating the average, since the hit-rate varies between different combination of the data set. In this work the average hit-rate is calculated from 10 runs, and then compared with the baseline method. 


\section{A. Clustering-based Tagging}

Since we have introduced two clustering algorithms in this method, the evaluation is conducted on both algorithms at the same time. For each run, the shuffled training data set and testing data set are the same to both clustering algorithms. By doing this, we can make sure that two algorithms are compared fairly. The hit-rate of two clustering algorithms and the baseline method is shown in Figure 4.

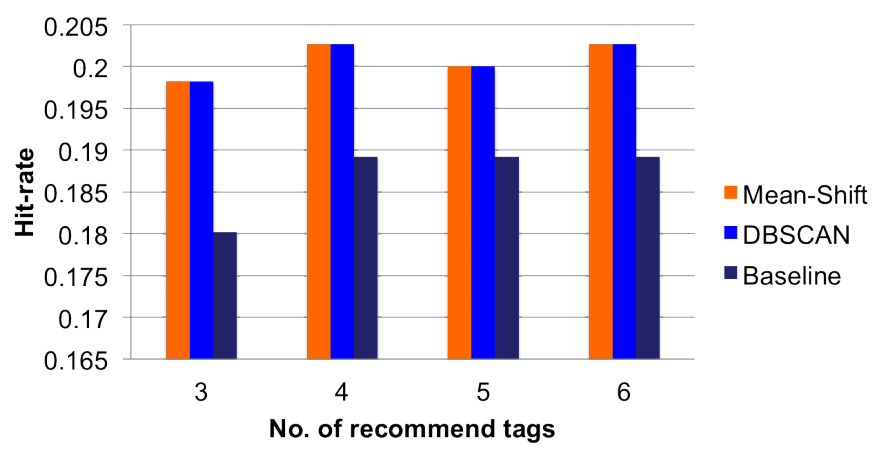

Fig. 4. Hit-rate of Clustering-based Tagging

We can see that the hit-rates of both algorithms are just a little bit higher than the baseline method. In addition, the hitrates of both algorithms are exactly the same. By analyzing the results, we found that both algorithms cluster the training data into two clusters, and one cluster has no location context (both SSID and GPS coordinates) or lack one of these location context. This may be caused by the limited number of photos we obtained. Since the location context contributes a major part of the feature, it can dominate the clustering result. In general, this approach can always recommend the tags of significant place name or event, but failed to recommend the content of a picture as expected.

While the results seem to be not so promising, it shows that we can leverage context to cluster photos and the hit-rate of this approach is better than the baseline method. We expect that if we can build a more comprehensive data set so more clusters can be found by the clustering algorithms, the hit-rate would be much higher.

\section{B. K-Neighbor Similarity Tagging}

In order to evaluate whether different context information has different influence on the hit-rate, we conduct the evaluation based on different combination of context. One is evaluated only using the location context. Considering there are few participants in our data set, the second evaluation uses all other context except the location context. The hit-rate is compared with the method using all context and the baseline method. Figure 5 shows the results.

The hit-rate of this approach is much better than both the baseline method and the clustering-based tagging method. We can see from the results that using the whole contextual information has the highest hit-rate, while using only location context is a little bit lower but still higher than non-location context. This result verifies that location context plays an important role in the recommendation. But does not dominate

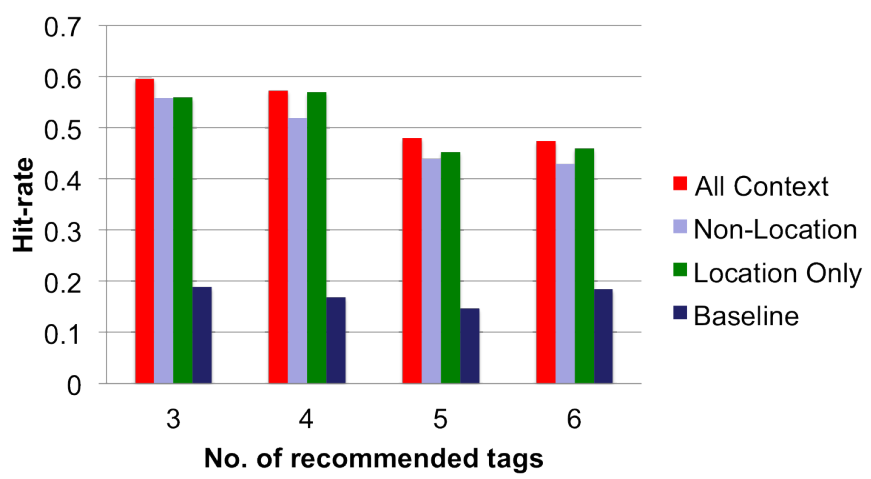

Fig. 5. K-Neighbor Similarity Tagging Hit-rate

the hit-rate, since adding more contextual information can increase the hit-rate.

The biggest advantage of this approach is that it works very well for one user, since the contextual information is consistent for one user. The disadvantage is that the hit-rate can be affected by some contextual information since we need to calculate the distance of two photos. For some photos, the temporal context may play an important role while the location context may play an important role for others. This approach cannot capture this variation. Hence, even if the distance of two photos is very close, the tags can be quite different.

\section{CONCLUSION}

We have presented our exploration of leveraging rich contextual information to automatically tag mobile photos. Our result verifies that there are intrinsic connections between contextual information and photos tags. The performance of both approaches we proposed exceeds the baseline method. The clustering-based tagging approach cannot cluster the photos well for a small data set, but it can still be used for autotagging. The K-Neighbor similarity tagging approach works very well for one user and its hit-rate is much higher than other approaches. Among all context we proposed, the location context plays a significant role both in clustering and autotagging procedures. In addition, other contextual information also has positive contribution since adding more context results in a higher hit-rate. In the future work, we plan to explore more context such as orientation, ambient light, and transportation mode.

\section{ACKNOWLEDGMENT}

This work is supported partly by the National Science Foundation under Grant No. 1040725 and No. 1016823. Any opinions, findings, and conclusions or recommendations expressed in this work are those of the author(s) and do not necessarily reflect the views of the National Science Foundation.

\section{REFERENCES}

[1] R. Datta, D. Joshi, J. Li, and J. Z. Wang, "Image retrieval: Ideas, influences, and trends of the new age," ACM Comput. Surv., vol. 40, no. 2, pp. 5:1-5:60, May 2008. [Online]. Available: http://doi.acm.org/10.1145/1348246.1348248 
[2] G. Carneiro, A. Chan, P. Moreno, and N. Vasconcelos, "Supervised learning of semantic classes for image annotation and retrieval," Pattern Analysis and Machine Intelligence, IEEE Transactions on, vol. 29, no. 3, pp. 394-410, 2007.

[3] R. Sarvas, E. Herrarte, A. Wilhelm, and M. Davis, "Metadata creation system for mobile images," in Proceedings of the 2nd international conference on Mobile systems, applications, and services, ser. MobiSys '04. New York, NY, USA: ACM, 2004, pp. 36-48. [Online]. Available: http://doi.acm.org/10.1145/990064.990072

[4] M. Davis, S. King, N. Good, and R. Sarvas, "From context to content: leveraging context to infer media metadata," in Proceedings of the 12th annual ACM international conference on Multimedia, ser. MULTIMEDIA '04. New York, NY, USA: ACM, 2004, pp. 188-195. [Online]. Available: http://doi.acm.org/10.1145/1027527.1027572

[5] M. Naaman, R. B. Yeh, H. Garcia-Molina, and A. Paepcke, "Leveraging context to resolve identity in photo albums," in Proceedings of the 5th ACM/IEEE-CS joint conference on Digital libraries, ser. JCDL '05. New York, NY, USA: ACM, 2005, pp. 178-187. [Online]. Available: http://doi.acm.org/10.1145/1065385.1065430

[6] C. Qin, X. Bao, R. Roy Choudhury, and S. Nelakuditi, "Tagsense: a smartphone-based approach to automatic image tagging," in Proceedings of the 9th international conference on Mobile systems, applications, and services, ser. MobiSys '11. New York, NY, USA: ACM, 2011, pp. 1-14. [Online]. Available: http://doi.acm.org/10.1145/1999995.1999997

[7] D. Comaniciu and P. Meer, "Mean shift: a robust approach toward feature space analysis," Pattern Analysis and Machine Intelligence, IEEE Transactions on, vol. 24, no. 5, pp. 603-619, 2002.

[8] M. Ester, H. peter Kriegel, J. S, and X. Xu, "A density-based algorithm for discovering clusters in large spatial databases with noise." AAAI Press, 1996, pp. 226-231. 